PROCEEDINGS OF THE

AMERICAN MATHEMATICAL SOCIETY

Volume 136, Number 9, September 2008, Pages 3309-3318

S 0002-9939(08)09304-0

Article electronically published on May 5, 2008

\title{
FIRST EIGENVALUE OF A JACOBI OPERATOR OF HYPERSURFACES WITH A CONSTANT SCALAR CURVATURE
}

\author{
QING-MING CHENG
}

(Communicated by Richard A. Wentworth)

\begin{abstract}
Let $M$ be an $n$-dimensional compact hypersurface with constant scalar curvature $n(n-1) r, r>1$, in a unit sphere $S^{n+1}(1)$. We know that such hypersurfaces can be characterized as critical points for a variational problem of the integral $\int_{M} H d M$ of the mean curvature $H$. In this paper, we first study the eigenvalue of the Jacobi operator $J_{s}$ of $M$. We derive an optimal upper bound for the first eigenvalue of $J_{s}$, and this bound is attained if and only if $M$ is a totally umbilical and non-totally geodesic hypersurface or $M$ is a Riemannian product $S^{m}(c) \times S^{n-m}\left(\sqrt{1-c^{2}}\right), 1 \leq m \leq n-1$.
\end{abstract}

\section{INTRODUCTION}

Let $M$ be an $n$-dimensional hypersurface in a unit sphere $S^{n+1}(1)$ of dimension $n+1$. We denote the second fundamental form of $M$ and its squared norm by $\alpha$ and S, respectively. Then, a Schrödinger operator

$$
J_{m}=-\Delta-S-n,
$$

where $\Delta$ stands for the Laplace-Beltrami operator, arose naturally in the study of the stability of both minimal hypersurfaces in $S^{n+1}(1)$ and hypersurfaces with constant mean curvature in $S^{n+1}(1)$. The $J_{m}$ is called a Jacobi operator or a stability operator, which represents the second variation of the volume. Its spectral behavior is directly related to the instability of such hypersurfaces (cf. [18] and [5]).

On the other hand, for any $C^{2}$-function $f$, denoting its Hessian by $\left(f_{i j}\right)$, we define a differential operator

$$
\square f=\sum_{i, j=1}^{n}\left(n H \delta_{i j}-h_{i j}\right) f_{i j}
$$

where $H$ and $h_{i j}$ denote the mean curvature and components of the second fundamental form of $M$. The differential operator $\square$ was introduced and used by S. Y. Cheng and Yau in 11 to study compact hypersurfaces with constant scalar

Received by the editors November 14, 2006, and, in revised form, August 2, 2007.

2000 Mathematics Subject Classification. Primary 53C42; Secondary 58J50.

Key words and phrases. Hypersurface with constant scalar curvature, Jacobi operator, mean curvature, first eigenvalue and principal curvatures.

The author's research was partially supported by a Grant-in-Aid for Scientific Research from JSPS. 
curvature in $S^{n+1}(1)$. They proved that if $M$ is an $n$-dimensional compact hypersurface with constant scalar curvature $n(n-1) r, r \geq 1$, and if the sectional curvature of $M$ is non-negative, then $M$ is a totally umbilical hypersurface $S^{n}(c)$ or a Riemannian product $S^{m}(c) \times S^{n-m}\left(\sqrt{1-c^{2}}\right), 1 \leq m \leq n-1$, where $S^{k}(c)$ denotes a sphere of radius $c$. We should notice that the differential operator $\square$ is self-adjoint. By making use of the similar method which has been used by Nakagawa and the author in 9 and the differential operator $\square$ introduced by S.Y. Cheng and Yau, Li 14 has proved that if $M$ is an $n$-dimensional compact hypersurface with constant scalar curvature $n(n-1) r, r \geq 1$, and if $S \leq(n-1) \frac{n(r-1)+2}{n-2}+\frac{n-2}{n(r-1)+2}$, then $M$ is a totally umbilical hypersurface or a Riemannian product $S^{n-1}(c) \times S^{1}\left(\sqrt{1-c^{2}}\right)$ with $c^{2}=\frac{n-2}{n r} \leq \frac{n-2}{n}$. Furthermore, the Riemannian product $S^{n-1}(c) \times S^{1}\left(\sqrt{1-c^{2}}\right)$ has been characterized in [6, 7], 8, and [10.

In 11, Alencar, do Carmo and Colares have studied the stability of hypersurfaces with constant scalar curvature in $S^{n+1}(1)$. In this case, the Jacobi operator $J_{s}$ is given by

$$
J_{s}=-\square-\left\{n(n-1) H+n H S-f_{3}\right\},
$$

which is associated to the variational characterization of hypersurfaces with constant scalar curvature in $S^{n+1}(1)$, where $f_{3}=\sum_{j=1}^{n} k_{j}^{3}$ and $k_{j}$ 's are the principal curvatures of $M$ (cf. [16] and [17]). The spectral behavior of $J_{s}$ is also directly related to the instability of hypersurfaces with constant scalar curvature.

The first eigenvalue of the Jacobi operator $J_{m}$ of minimal hypersurfaces in $S^{n+1}(1)$ was studied by Simons [18, Wu [19] and Perdomo [15]. A characterization of the Clifford torus is given by the first eigenvalue of the Jacobi operator $J_{m}$, that is, they proved that if $M$ is an $n$-dimensional compact orientable minimal hypersurface in $S^{n+1}(1)$, then the first eigenvalue $\lambda_{1}^{J_{m}}$ of the Jacobi operator $J_{m}$ satisfies

(1) $\lambda_{1}^{J_{m}}=-n$ and $M$ is totally geodesic.

(2) $\lambda_{1}^{J_{m}} \leq-2 n$ and $\lambda_{1}^{J_{m}}=-2 n$ if and only if $M$ is a Clifford torus $S^{m}\left(\sqrt{\frac{m}{n}}\right) \times$ $S^{n-m}\left(\sqrt{\frac{n-m}{n}}\right), 1 \leq m \leq n-1$.

Very recently, Alías, Barros and Brasil [3] have extended the above results to compact hypersurfaces with constant mean curvature in $S^{n+1}(1)$. They have obtained that if $M$ is an $n$-dimensional compact orientable hypersurface with constant mean curvature in $S^{n+1}(1)$, then the first eigenvalue $\lambda_{1}^{J_{m}}$ of the Jacobi operator $J_{m}$ satisfies

(1) $\lambda_{1}^{J_{m}}=-n\left(1+H^{2}\right)$ and $M$ is totally umbilical or

(2) $\lambda_{1}^{J_{m}} \leq-2 n\left(1+H^{2}\right)+\frac{n(n-2)}{\sqrt{n(n-1)}}|H| \max \sqrt{S-n H^{2}}$ and equality holds if and only if $M$ is a Riemannian product $S^{n-1}(c) \times S^{1}\left(\sqrt{1-c^{2}}\right)$.

We should notice that the first eigenvalue of the Jacobi operator $J_{m}$ of the totally umbilical hypersurface $S^{n+1}(1)$ is different from the one of the Riemannian product $S^{n-1}(c) \times S^{1}\left(\sqrt{1-c^{2}}\right)$ in $S^{n+1}(1)$.

Since the Laplace-Beltrami operator is always elliptic, the Jacobi operator $J_{m}$ is always elliptic. But, in general, the operator $\square$, and hence the Jacobi operator $J_{s}$, are not elliptic. When $r>1$, the differential operator $\square$ is elliptic. In fact, from the Gauss equation (2.4) in section 2, we have that the mean curvature $H$ satisfies $n^{2} H^{2}>S$. Hence, we can assume $H>0$. Thus, the differential operator $\square$ is 
elliptic if and only if $n H-k_{j}>0$ for $j=1,2, \cdots, n$, where $k_{j}$ 's are the principal curvatures of $M$. If, for some $j, n H \leq k_{j}$ holds, then $n^{2} H^{2} \leq k_{j}^{2} \leq S$. This is impossible.

When $r=1$, let $k_{i}, i=1,2, \cdots, n$, denote the principal curvatures of $M$. We consider the elementary symmetric functions $S_{r}$ of the principal curvatures

$$
S_{0}=1, \quad S_{r}=\sum_{i_{1}<\cdots<i_{r}} k_{i_{1}} \cdots k_{i_{r}} \quad(1 \leq r \leq n) .
$$

is elliptic if and only if $n \geq 3$ and the $S_{3} \neq 0$ on $M$ (cf. [4, [2] and [12]).

In [4, Alías, Brasil and Sousa have studied the first eigenvalue of the Jacobi operator $J_{s}$ of an $n$-dimensional hypersurface with constant scalar curvature $n(n-1)$. They have proved that if $M$ is an $n$-dimensional compact orientable hypersurface with constant scalar curvature $n(n-1)$ in $S^{n+1}(1)$ and if $n \geq 3$ and $S_{3}$ does not vanish on $M$, then the first eigenvalue $\lambda_{1}^{J_{s}}$ of the Jacobi operator $J_{s}$ satisfies

$$
\lambda_{1}^{J_{s}} \leq-2 n(n-1) \min |H| .
$$

Further, the equality holds if and only if $M$ is a Riemannian product $S^{m}(c) \times$ $S^{n-m}\left(\sqrt{1-c^{2}}\right), 1 \leq m \leq n-1$, with scalar curvature $n(n-1)$.

In this paper, we investigate the first eigenvalue of the Jacobi operator $J_{s}$ of an $n$ dimensional hypersurface with constant scalar curvature $n(n-1) r, r>1$, and give a characterization of the totally umbilical and non-totally geodesic hypersurface and the Riemannian product $S^{m}(c) \times S^{n-m}\left(\sqrt{1-c^{2}}\right), 1 \leq m \leq n-1$, by the first eigenvalue of $J_{s}$. Namely, we will prove the following:

Theorem 1.1. Let $M$ be an n-dimensional compact orientable hypersurface with constant scalar curvature $n(n-1) r, r>1$, in $S^{n+1}(1)$. Then, the Jacobi operator $J_{s}$ is elliptic, the mean curvature $H$ does not vanish on $M$ and the first eigenvalue $\lambda_{1}^{J_{s}}$ of the Jacobi operator $J_{s}$ satisfies

$\lambda_{1}^{J_{s}} \leq-\left\{2 n(n-1)+n^{2}(n-1)(r-1)\right\} \min |H|+n(n-1)(r-1)\{(n-1)(r-1)+1\} \frac{1}{\min |H|}$

and the equality holds if and only if either $M$ is totally umbilical and non-totally geodesic or $M$ is a Riemannian product $S^{m}(c) \times S^{n-m}\left(\sqrt{1-c^{2}}\right), 1 \leq m \leq n-1$, with $r>1$.

Corollary 1.2. Let $M$ be an n-dimensional compact orientable hypersurface with constant scalar curvature $n(n-1) r, r>1$, in $S^{n+1}(1)$. Then, the Jacobi operator $J_{s}$ is elliptic and the first eigenvalue $\lambda_{1}^{J_{s}}$ of the Jacobi operator $J_{s}$ satisfies

$$
\lambda_{1}^{J_{s}} \leq-n(n-1) r \sqrt{r-1}
$$

and the equality holds if and only if $M$ is totally umbilical and non-totally geodesic.

Remark 1.3. We should notice that the totally umbilical hypersurfaces do not appear in the result of [4. From the proof of Theorem 1.1 in section 3, we shall see that our results do hold for the case where $r=1$, if we assume that the Jacobi operator $J_{s}$ is elliptic. Hence, the result in 4 can be seen as a direct consequence of Theorem 1.1. 


\section{Preliminary}

Throughout this paper, all manifolds are assumed to be smooth and connected without boundary. Let $M$ be an $n$-dimensional hypersurface in a unit sphere $S^{n+1}(1)$. We choose a local orthonormal frame $\left\{\mathbf{e}_{1}, \cdots, \mathbf{e}_{n}, \mathbf{e}_{n+1}\right\}$ and the dual coframe $\left\{\omega_{1}, \cdots, \omega_{n}, \omega_{n+1}\right\}$ in such a way that $\left\{\mathbf{e}_{1}, \cdots, \mathbf{e}_{n}\right\}$ is a local orthonormal frame on $M$. Hence, we have

$$
\omega_{n+1}=0
$$

on $M$. From Cartan's lemma, we have

$$
\omega_{i n+1}=\sum_{j=1}^{n} h_{i j} \omega_{j}, h_{i j}=h_{j i} .
$$

The mean curvature $H$ and the second fundamental form $\alpha$ of $M$ are defined, respectively, by

$$
H=\frac{1}{n} \sum_{i=1}^{n} h_{i i}, \alpha=\sum_{i, j=1}^{n} h_{i j} \omega_{i} \otimes \omega_{j} \mathbf{e}_{n+1} .
$$

When the mean curvature $H$ of $M$ is identically zero, we recall that $M$ is by definition a minimal hypersurface.

From the structure equations of $M$, the Gauss equation and the Codazzi equation are given by

$$
\begin{gathered}
R_{i j k l}=\left(\delta_{i k} \delta_{j l}-\delta_{i l} \delta_{j k}\right)+\left(h_{i k} h_{j l}-h_{i l} h_{j k}\right), \\
h_{i j k}=h_{i k j} .
\end{gathered}
$$

From (2.2), we have

$$
n(n-1) r=n(n-1)+n^{2} H^{2}-S,
$$

where $n(n-1) r$ and $S$ denote the scalar curvature and the squared norm of the second fundamental form of $M$, respectively.

For any $C^{2}$-function $f$ on $M$, we define its gradient and Hessian by

$$
\begin{gathered}
d f=\sum_{i=1}^{n} f_{i} \omega_{i} \\
\sum_{j=1}^{n} f_{i j} \omega_{j}=d f_{i}+\sum_{j=1}^{n} f_{j} \omega_{j i} .
\end{gathered}
$$

Thus, the differential operator $\square$ is defined by

$$
\square f=\sum_{i, j=1}^{n}\left(n H \delta_{i j}-h_{i j}\right) f_{i j}
$$




\section{Proofs of Results}

First of all, we will consider the first eigenvalue of the Jacobi operator $J_{s}$ of both the totally umbilical and non-totally geodesic hypersurface and the Riemannian product $S^{m}(c) \times S^{n-m}\left(\sqrt{1-c^{2}}\right), 1 \leq m \leq n-1$.

Example 3.1. Let $M$ be a totally umbilical and non-totally geodesic hypersurface in $S^{n+1}(1)$. In this case, $\square=(n-1) H \Delta$ and

$$
J_{s}=-\square-\left\{n(n-1) H+n H S-f_{3}\right\}=-\left\{(n-1) H \Delta+n(n-1) H\left(1+H^{2}\right)\right\} .
$$

Hence,

$$
\lambda_{1}^{J_{s}}=-n(n-1) H\left(1+H^{2}\right)=-n(n-1) r \sqrt{r-1},
$$

from the Gauss equation (2.4). Since

$$
\begin{aligned}
& n(n-1) r \sqrt{r-1} \\
= & \left\{2 n(n-1)+n^{2}(n-1)(r-1)\right\} H-n(n-1)(r-1)\{(n-1)(r-1)+1\} \frac{1}{H},
\end{aligned}
$$

we know that

$$
\begin{aligned}
\lambda_{1}^{J_{s}} & =n(n-1) r \sqrt{r-1} \\
& =-\left\{2 n(n-1)+n^{2}(n-1)(r-1)\right\} H \\
& +n(n-1)(r-1)\{(n-1)(r-1)+1\} \frac{1}{H} .
\end{aligned}
$$

Example 3.2. Let $S^{m}(c) \times S^{n-m}\left(\sqrt{1-c^{2}}\right), 1 \leq m \leq n-1$, be a hypersurface with $r>1$ in $S^{n+1}(1)$. In this case, the position vector is $\mathbf{x}=\left(\mathbf{x}_{1}, \mathbf{x}_{2}\right) \in S^{m}(c) \times$ $S^{n-m}\left(\sqrt{1-c^{2}}\right)$ and the unit normal vector at the point $\mathbf{x}=\left(\mathbf{x}_{1}, \mathbf{x}_{2}\right) \in S^{m}(c) \times$ $S^{n-m}\left(\sqrt{1-c^{2}}\right)$ is given by $\mathbf{e}_{n+1}=\left(-\frac{\sqrt{1-c^{2}}}{c} \mathbf{x}_{1}, \frac{c}{\sqrt{1-c^{2}}} \mathbf{x}_{2}\right)$. Its principal curvatures are given by

$$
k_{1}=\cdots=k_{m}=\frac{\sqrt{1-c^{2}}}{c}, \quad k_{m+1}=\cdots=k_{n}=-\frac{c}{\sqrt{1-c^{2}}} .
$$

Thus, the mean curvature $H$ of $S^{m}(c) \times S^{n-m}\left(\sqrt{1-c^{2}}\right)$ satisfies

$$
n H=\frac{m-n c^{2}}{c \sqrt{1-c^{2}}} \text {. }
$$

Since

$$
\begin{aligned}
& n(n-1)(r-1)=n^{2} H^{2}-S \\
& =\frac{\left(m-n c^{2}\right)^{2}}{c^{2}\left(1-c^{2}\right)}-m \frac{1-c^{2}}{c^{2}}-(n-m) \frac{c^{2}}{1-c^{2}} \\
& =\frac{n(n-1) c^{4}-2 m(n-1) c^{2}+m(m-1)}{c^{2}\left(1-c^{2}\right)} \\
& =\frac{(n-1)\left(n c^{2}+n-2 m\right)\left(c^{2}-1\right)+(n-m)(n-1-m)}{c^{2}\left(1-c^{2}\right)},
\end{aligned}
$$


we know that $r>1$ if and only if either

$$
0<c^{2}<\frac{m}{n}-\frac{\sqrt{m(n-m)}}{n \sqrt{n-1}}
$$

or

$$
\frac{m}{n}+\frac{\sqrt{m(n-m)}}{n \sqrt{n-1}}<c^{2}<1 .
$$

Since the principal curvatures of $S^{m}(c) \times S^{n-m}\left(\sqrt{1-c^{2}}\right)$ are constant, we know that $S$ and $f_{3}$ are constant. Because the differential operator $\square$ is self-adjoint and elliptic, we have that the first eigenvalue of the Jacobi operator $J_{s}$ is given by

$$
\lambda_{1}^{J_{s}}=-\left\{n(n-1) H+n H S-f_{3}\right\} .
$$

Furthermore, from (3.1), we have

$$
\begin{aligned}
& n(n-1) H+n H S-f_{3} \\
= & n(n-1) H+n H\left\{m \frac{1-c^{2}}{c^{2}}+(n-m) \frac{c^{2}}{1-c^{2}}\right\} \\
& -m \frac{\left(1-c^{2}\right)^{\frac{3}{2}}}{c^{3}}+(n-m) \frac{c^{3}}{\left(1-c^{2}\right)^{\frac{3}{2}}} \\
= & n H\left\{n-1+m \frac{1-c^{2}}{c^{2}}+(n-m) \frac{c^{2}}{1-c^{2}}\right. \\
& \left.-\frac{m}{m-n c^{2}} \frac{\left(1-c^{2}\right)^{2}}{c^{2}}+\frac{(n-m)}{m-n c^{2}} \frac{c^{4}}{\left(1-c^{2}\right)}\right\} \\
= & \frac{n H}{m-n c^{2}}\left\{(n-1)\left(m-n c^{2}\right)+\frac{m\left(1-c^{2}\right)\left\{m-1-(n-1) c^{2}\right\}}{c^{2}}\right. \\
& \left.+\frac{(n-m) c^{2}\left\{m-(n-1) c^{2}\right\}}{1-c^{2}}\right\} .
\end{aligned}
$$

On the other hand, from (3.1) and (3.2), we have

$$
\begin{aligned}
& n(n-1)(r-1) \frac{1}{n^{2} H^{2}} \\
& =\frac{(n-1)\left(n c^{2}+n-2 m\right)\left(c^{2}-1\right)+(n-m)(n-1-m)}{\left(m-n c^{2}\right)^{2}}, \\
& \left\{2 n(n-1)+n^{2}(n-1)(r-1)\right\} H \\
& =n H \frac{(n-1)\left\{(n-2) c^{2}+n-2 m\right\}\left(c^{2}-1\right)+(n-m)(n-1-m)}{c^{2}\left(1-c^{2}\right)} .
\end{aligned}
$$


Therefore, we obtain, from (3.2), (3.5) and (3.6), by making use of a direct computation,

$$
\begin{aligned}
& \left\{2 n(n-1)+n^{2}(n-1)(r-1)\right\} H \\
& -n(n-1)(r-1)\{(n-1)(r-1)+1\} \frac{1}{H} \\
& =n H \frac{(n-1)\left\{(n-2) c^{2}+n-2 m\right\}\left(c^{2}-1\right)+(n-m)(n-1-m)}{c^{2}\left(1-c^{2}\right)} \\
& -n H \frac{(n-1)\left(n c^{2}+n-2 m\right)\left(c^{2}-1\right)+(n-m)(n-1-m)}{\left(m-n c^{2}\right)^{2}} \\
& \times \frac{\left\{n(n-2) c^{2}+(n-1)(n-2 m)\right\}\left(c^{2}-1\right)+(n-m)(n-1-m)}{c^{2}\left(1-c^{2}\right)} \\
& =\frac{n H}{\left(m-n c^{2}\right)^{2} c^{2}\left(1-c^{2}\right)}\left\{2(n-1) c^{2}\left(1-c^{2}\right)\left(m-n c^{2}\right)^{2}\right. \\
& +\left\{(n-1)\left(n c^{2}+n-2 m\right)\left(c^{2}-1\right)+(n-m)(n-1-m)\right\} \\
& \times\left[\left(m-n c^{2}\right)^{2}-\left[\left\{n(n-2) c^{2}+(n-1)(n-2 m)\right\}\left(c^{2}-1\right)\right.\right. \\
& +(n-m)(n-1-m)]]\} \\
& =\frac{n H}{\left(m-n c^{2}\right)^{2} c^{2}\left(1-c^{2}\right)}\left\{2(n-1) c^{2}\left(1-c^{2}\right)\left(m-n c^{2}\right)^{2}\right. \\
& \left.+\left[(n-1)\left(n c^{2}+n-2 m\right)\left(c^{2}-1\right)+(n-m)(n-1-m)\right]\left(m-n c^{2}\right)\left(1-2 c^{2}\right)\right\} \\
& =\frac{n H}{\left(m-n c^{2}\right) c^{2}\left(1-c^{2}\right)}\left\{2(n-1) c^{2}\left(1-c^{2}\right)\left(m-n c^{2}\right)\right. \\
& \left.+(n-1)\left(n c^{2}-m\right)\left(c^{2}-1\right)\left(1-2 c^{2}\right)+(n-m)\left\{(n-1) c^{2}-m\right\}\left(1-2 c^{2}\right)\right\} \\
& =\frac{n H}{\left(m-n c^{2}\right)}\left\{\frac{(n-1)\left(m-n c^{2}\right)}{c^{2}}\right. \\
& \left.+\frac{(n-m)\left\{(n-1) c^{2}-m\right\}}{c^{2}}+\frac{(n-m)\left\{m-(n-1) c^{2}\right\}}{1-c^{2}}\right\} \\
& =\frac{n H}{m-n c^{2}}\left\{(n-1)\left(m-n c^{2}\right)+\frac{m\left(1-c^{2}\right)\left\{m-1-(n-1) c^{2}\right\}}{c^{2}}\right. \\
& \left.+\frac{(n-m) c^{2}\left\{m-(n-1) c^{2}\right\}}{1-c^{2}}\right\} \text {. }
\end{aligned}
$$

Hence, we infer, from (3.4) and the above equality, that

$$
\begin{aligned}
\lambda_{1}^{J_{s}}= & -\left\{n(n-1) H+n H S-f_{3}\right\} \\
= & -\left\{2 n(n-1)+n^{2}(n-1)(r-1)\right\} H \\
& +n(n-1)(r-1)\{(n-1)(r-1)+1\} \frac{1}{H} .
\end{aligned}
$$


Remark 3.3. We must notice that the first eigenvalue of the Jacobi operator $J_{s}$ of both the totally umbilical and non-totally geodesic hypersurfaces and the Riemannian product $S^{m}(c) \times S^{n-m}\left(\sqrt{1-c^{2}}\right)$ has the same representation formula. But, as we have seen in the introduction, the first eigenvalue of the Jacobi operator $J_{m}$ of the totally umbilical hypersurface is different from the first eigenvalue of the Jacobi operator $J_{m}$ of the Riemannian product $S^{n-1}(c) \times S^{1}\left(\sqrt{1-c^{2}}\right)$. It is a very interesting fact.

Proof of Theorem 1.1. Since $r>1$, from the assertion in the introduction, we know that $\square$ is elliptic and $n^{2} H^{2}-S=n(n-1)(r-1)>0$. Hence, $H \neq 0$. Thus, we can assume $H>0$. We choose a local field of orthonormal frames $\mathbf{e}_{1}, \cdots, \mathbf{e}_{n}$ on $M$ such that, at the point that we consider,

$$
h_{i j}=\left\{\begin{aligned}
k_{i} & \text { if } \quad i=j \\
0 & \text { if } \quad i \neq j
\end{aligned}\right.
$$

where the $k_{i}$ 's are the principal curvatures of $M$. Thus,

$$
\square(n H)=n H \Delta(n H)-\sum_{i=1}^{n} k_{i}(n H)_{i i} .
$$

From the Gauss equation (2.4), we have

$$
n H \Delta(n H)=\frac{1}{2} \Delta(n H)^{2}-|\nabla(n H)|^{2}=\frac{1}{2} \Delta S-|\nabla(n H)|^{2} .
$$

By making use of a standard computation of Simons' type formula (cf. [11, 1] and [18]), we have

$$
\frac{1}{2} \Delta S=\sum_{i, j, k=1}^{n} h_{i j k}^{2}+\sum_{i=1}^{n} k_{i}(n H)_{i i}+n S-n^{2} H^{2}+n H f_{3}-S^{2} .
$$

Hence, we infer

$$
\square(n H)=\sum_{i, j, k=1}^{n} h_{i j k}^{2}-n^{2}|\nabla H|^{2}+n S-n^{2} H^{2}+n H f_{3}-S^{2} .
$$

Therefore, we have

$$
\begin{aligned}
J_{s} H= & -\square H-\left\{n(n-1) H+n H S-f_{3}\right\} H \\
= & -\left\{\frac{1}{n} \sum_{i, j, k=1}^{n} h_{i j k}^{2}-n|\nabla H|^{2}+S-n H^{2}+H f_{3}\right. \\
& \left.-\frac{S^{2}}{n}+n(n-1) H^{2}+n H^{2} S-H f_{3}\right\} \\
= & -\left\{\frac{1}{n} \sum_{i, j, k=1}^{n} h_{i j k}^{2}-n|\nabla H|^{2}+S+n(n-2) H^{2}+n H^{2} S-\frac{S^{2}}{n}\right\} \\
= & -\left[\frac{1}{n} \sum_{i, j, k=1}^{n} h_{i j k}^{2}-n|\nabla H|^{2}+2 n(n-1) H^{2}\right. \\
& \left.+n^{2}(n-1)(r-1) H^{2}-n(n-1)(r-1)\{(n-1)(r-1)+1\}\right] .
\end{aligned}
$$


From the min-max principle, we have

$$
\begin{aligned}
\lambda_{1}^{J_{s}} \leq & \frac{\int_{M} H J_{s} H d M}{\int_{M} H^{2} d M} \\
= & -\frac{\int_{M} H\left(\frac{1}{n} \sum_{i, j, k=1}^{n} h_{i j k}^{2}-n|\nabla H|^{2}\right) d M}{\int_{M} H^{2} d M} \\
& -\frac{\int_{M} H\left\{2 n(n-1)+n^{2}(n-1)(r-1)\right\} H^{2} d M}{\int_{M} H^{2} d M} \\
& +\frac{\int_{M} H n(n-1)(r-1)\{(n-1)(r-1)+1\} d M}{\int_{M} H^{2} d M} \\
\leq & -\left\{2 n(n-1)+n^{2}(n-1)(r-1)\right\} \min |H| \\
& +n(n-1)(r-1)\{(n-1)(r-1)+1\} \frac{1}{\min |H|} .
\end{aligned}
$$

Here we have used the following inequality (cf. [1] and [14]):

$$
\sum_{i, j, k=1}^{n} h_{i j k}^{2} \geq n^{2}|\nabla H|^{2}
$$

which can be proved by using the Gauss equation (2.4) and $r>1$. When the equality holds, we know that $H$ is constant and $\sum_{i, j, k=1}^{n} h_{i j k}^{2}=0$ on $M$, that is, the second fundamental form of $M$ is parallel. Hence, $M$ is an isoparametric hypersurface with at most two distinct principal curvatures (cf. [13]). Thus, $M$ is totally umbilical and non-totally geodesic or a Riemannian product $S^{m}(c) \times$ $S^{n-m}\left(\sqrt{1-c^{2}}\right), 1 \leq m \leq n-1$.

Proof of Corollary 1.2. From the Gauss equation (2.4) and $r>1$, we have $n(n-1) H^{2} \geq n^{2} H^{2}-S=n(n-1)(r-1)>0$. Hence, $|H| \geq \sqrt{r-1}$ and the equality holds at umbilical points. Since $|H| \geq \sqrt{r-1}>0$, we have $H \neq 0$. From $|H| \geq \sqrt{r-1}$, we infer

$$
\begin{aligned}
& -\left\{2 n(n-1)+n^{2}(n-1)(r-1)\right\} \min |H| \\
& +n(n-1)(r-1)\{(n-1)(r-1)+1\} \frac{1}{\min |H|} \\
& \leq-n(n-1) r \min |H| \leq-n(n-1) r \sqrt{r-1} .
\end{aligned}
$$

Therefore, we know that the first eigenvalue of the Jacobi operator $J_{s}$ satisfies $\lambda_{1}^{J_{s}} \leq-n(n-1) r \sqrt{r-1}$. When equality holds, we know

$$
\begin{aligned}
\lambda_{1}^{J_{s}}= & -\left\{2 n(n-1)+n^{2}(n-1)(r-1)\right\} \min |H| \\
& +n(n-1)(r-1)\{(n-1)(r-1)+1\} \frac{1}{\min |H|} \\
= & -n(n-1) r \sqrt{r-1} .
\end{aligned}
$$

Hence, $H$ is constant from Theorem 1.1 and $S=n H^{2}$. Namely, $M$ is totally umbilical. If $M$ is totally umbilical, from Example 3.1, we know that $\lambda_{1}^{J_{s}}=$ $-n(n-1) r \sqrt{r-1}$. 


\section{ACKNOWLEDGEMENTS}

We would like to express our gratitude to the referee for introducing us to paper [4] and for valuable suggestions.

\section{REFERENCES}

1. Alencar, H., do Carmo, M., and Colares, A. G., Stable hypersurfaces with constant scalar curvature, Math. Z., 213(1993), 117-131. MR.1217674 (94d:53080)

2. Alencar, H., do Carmo, M., and Santos, W., A gap theorem for hypersurfaces of the sphere with constant scalar curvature one, Comment. Math. Helv., 77(2002), 549-562. MR.1933789 (2003m:53098)

3. Alías, L. J., Barros, A., and Brasil, A., Jr., A spectral characterization of $H(r)$-torus by the first stability eigenvalue, Proc. Amer. Math. Soc., 133(2005), 875-884. MR2113939 (2005j:53061)

4. Alías, L. J., Brasil, A., Jr., and Sousa, L., Jr., A characterization of Clifford tori with constant scalar curvatrue one by the first stability eigenvalue, Bull. Braz. Math. Soc., 35(2004), 165175. MR.2081021 (2005e:53089)

5. Barbosa, J. L., do Carmo, M., and Eschenburg, J., Stability of hypersurfaces with constant mean curvature in Riemannian manifolds, Math. Z., 197(1988), 123-138. MR917854 (88m:53109)

6. Cheng, Q.-M., The rigidity of Clifford torus $S^{1}\left(\sqrt{\frac{1}{n}}\right) \times S^{n-1}\left(\sqrt{\frac{n-1}{n}}\right)$, Comment. Math. Helv., 71(1996), 60-69. MR1371678(97a:53094)

7. Cheng, Q.-M., Hypersurfaces in a unit sphere $S^{n+1}(1)$ with constant scalar curvature, J. London Math. Soc., 64(2001), 755-768. MR1865560 (2002k:53116)

8. Cheng, Q.-M., Compact hypersurfaces in a unit sphere with infinite fundamental group, Pacific J. Math., 212(2003), 49-56. MR2016567(2004g:53059)

9. Cheng, Q.-M., and Nakagawa, H., Totally umbilical hypersurfaces, Hiroshima Math. J., 20(1990), 1-10. MR1050421 (91f:53054)

10. Cheng, Q.-M., Shu, S. C., and Suh, Y. J., Compact hypersurfaces in a unit sphere, Proc. Royal Soc. Edinburgh, 135A(2005), 1129-1137. MR2191892 (2006g:53074)

11. Cheng, S. Y., and Yau, S. T., Hypersurfaces with constant scalar curvature, Math. Ann., 225(1997), 195-204. MR0431043 (55:4045)

12. Hounie, J., and Leite, M. L., Two-ended hypersurfaces with zero scalar curvature, Indiana Univ. Math. J., 48(1999), 867-882. MR1736975 (2001b:53077)

13. Lawson, H. B., Jr., Local rigidity theorems for minimal hypersurfaces, Ann. of Math., 89 (1969), 167-179. MR0238229 (38:6505)

14. Li, H., Hypersurfaces with constant scalar curvature in space forms, Math. Ann., 305(1996), 665-672. MR 1399710 (97i:53073)

15. Perdomo, O., First stability eigenvalue characterization of Clifford hypersurfaces, Proc. Amer. Math. Soc., 130(2002), 3379-3384. MR1913017 (2003f:53109)

16. Reilly, R. C., Variational properties of functions of the mean curvatures for hypersurfaces in space forms, J. Diff. Geom., 8(1973), 465-477. MR0341351 (49:6102)

17. Rosenberg, H., Hypersurfaces of constant curvatures in space forms, Bull. Sci. Math., 117(1993), 211-239. MR.1216008 (94b:53097)

18. Simons, J., Minimal varieties in Riemannian manifolds, Ann. of Math., 88(1968), 62-105. MR 0233295 (38:1617)

19. Wu, C., New characterizations of the Clifford tori and the Veronese surface, Arch. Math. (Basel), 61(1993), 277-284. MR.1231163(94h:53084)

Department of Mathematics, Faculty of Science and Engineering, Saga University, SAGA 840-8502, JAPAN

E-mail address: cheng@ms.saga-u.ac.jp 